\title{
Performance Evaluation of Adaptive Continuous Wavelet Transform based Rake Receiver for UWB Systems
}

\author{
Ch. Navitha, K. Sivani, K. Ashoka Reddy \\ Dept. of Electronics \& Communication Engineering, Kakatiya Institute of Technology \& Science, \\ Warangal, Telangana, India
}

\begin{tabular}{l} 
Article Info \\
\hline Article history: \\
Received Apr 25, 2018 \\
Revised Jun 27, 2018 \\
Accepted Jul 4, 2018 \\
\hline
\end{tabular}

Keyword:

Adaptive rake receiver Channel impulse response Maximal ratio combining Rake receiver UWB systems Wavelet rake receiver

\begin{abstract}
This paper proposes an adaptive continuous wavelet transform (ACWT) based Rake receiver to mitigate interference for high speed ultra wideband (UWB) transmission. The major parts of the receiver are least mean square (LMS) adaptive equalizer and $\mathrm{N}$-selective maximum ratio combiner (MRC). The main advantage of using continuous wavelet rake receiver is that it utilizes the maximum bandwidth $(7.5 \mathrm{GHz})$ of the UWB transmitted signal, as announced by the Federal Communication Commission (FCC). In the proposed ACWT Rake receiver, the weights and the finger positions are updated depending upon the convergence error over a period in which training data is transmitted. Line of sight (LOS) channel model (CM1 from 0 to 4 meters) and the Non line of sight (NLOS) channel models (CM, CM3 and CM4) are the indoor channel models selected for investigating in this research . The performance of the proposed adaptive system is evaluated by comparing with conventional rake and continuous wavelet transform (CWT) based rake. It showed an improved performance in all the different UWB channels (CM1 to CM4) for rake fingers of 2, 4 and 8. Simulations showed that for 8 rake fingers, the proposed adaptive CWT rake receiver has shown an SNR improvement of $2 \mathrm{~dB}, 3 \mathrm{~dB}, 10 \mathrm{~dB}$ and $2 \mathrm{~dB}$ respectively over CWT rake receiver in different UWB channels CM1, CM2, CM3 and CM4.
\end{abstract}

Copyright $@ 2018$ Institute of Advanced Engineering and Science. All rights reserved.

\section{Corresponding Author:}

K. Ashoka Reddy,

Departement of Electronics \& Communication Engineering,

Kakatiya Institute of Technology \& Science,

Warangal, Telangana-506015, India.

Email: kareddy.iitm@gmail.com

\section{INTRODUCTION}

In recent years, ultra wideband (UWB) technologies have drawn great interest in the wireless community [1], [2].Among various applications, one of the most promising is in wireless sensor networks (WSNs) [3], [4] .UWB systems have potentially low complexity and low cost, with noise-like signal properties that create little interference to other systems, are resistant to severe multipath and jamming, and have very good time domain resolution allowing for precise location and tracking.

UWB system transmits very short pulses with relatively huge bandwidth [5]. UWB transmitted pulses have lower power of $-10 \mathrm{~dB}$ and bandwidth of order of several gigahertz's [6]. The use of this huge bandwidth accounts both advantages and disadvantages. The major advantage of this huge bandwidth is found when it is used in conjunction with the spreading techniques [7]-[13] provides robustness to jamming, as well as a low probability of detection. The major disadvantage of the UWB system due to its huge bandwidth is it always co-exists with the narrow band system or wide band system which already had dedicated frequency allocation in that band which results in high multiple signal interference [14].

Due to this dense multipath environment received signal contains many delayed and scaled replicas of the transmitted pulses. As most of the energy is distributed in these multipath components which can be 
captured and thus enhance the performance by using rake receiver that introduces the multipath diversity. In order to capture most of the energy distributed over these multipath components large numbers of rake fingers are to be used. Rake figures compose of weights, correlators with delayed versions of the transmitted pulse and template waveforms. These weights, delays will be the additions channel parameters that need to be estimated [15], [16].

A large number of research papers were presented on the Rake receiver [17] which also includes the study of the performance of the partial, chip, or symbol delays spacing Rake with channel estimator [18]. Its execution was additionally studied with two combining strategies the MRC and SLC (Square Law Combiner) in [19]. Likewise a Selective Rake (SRake) receiver which tracks the strongest L multipath components is proposed in [20] with its execution exhibited. In [21] the performance for UWB communication systems using different optimal model techniques in a RAKE receiver is investigated.

The wavelet transform (WT) technique is a modern area of mathematics that is applied for compressing signals and images and removing noise from their coefficients [22]. The signal-to noise ratio (SNR) can be improved by using a WT approach that decomposes the signal into different scales and different levels of resolution. Wavelet video compression was evaluated and achieved in [23], [24] for wideband, multi-carrier, code- division, multiple access (MC-CDMA) and a rake receiver over additive, white Gaussian noise (AWGN) and the Rayleigh fading channel. A novel wavelet rake receiver (WR) based on continuous wavelet transform (CWT) was presented in [25], and it showed enhancement in performance with a less complex receiver.

In [26], [27], wiener filters were used to estimate the channel. This approach of channel estimation is known as minimum mean square error (MMSE) solution, which provides more reliable estimation in fast fading channel. However to calculate the optimal wiener filter coefficients, require matrix inversion calculation which is highly complex. In [28] a linear prediction filter was introduces which is completely based on decision- feedback mode. This method overcomes the computational complexity introduced by the wiener filter approach.

In this paper, an adaptive continuous wavelet transforms (ACWT) based rake receiver is proposed and implemented where the finger gains and the delays are updated over the training period. LMS algorithm is used for the updating delays and gains of the finger.

\section{SYSTEM MODEL}

Two popular modulation types being considered for UWB are pulse-position modulation (PPM) and pulse amplitude modulation (PAM). Here, we consider systems of the PAM type, and a model for single-user PAM UWB transmission over channels with ISI. We have assumed a baseband transmission system, and hence all signals are assumed to be real-valued. The zero-mean independent and identically distributed(i.i.d) data symbols $\left\{s_{n}\right\}$ are passed through a unit energy pulse shaping filter $\mathrm{p}(\mathrm{t})$ which includes the effects of the transmit antenna. Note that we require the pulse shape to be unchanging from symbol period to symbol period, but our model is general enough to include either time hopping (TH) or direct sequence (DS) block spreading if, for example, $\mathrm{p}(\mathrm{t})$ is the sum of several delayed Gaussian monocycles.

Pulse waveform generation is a key technology in UWB system. There are some good candidates, such as Hermite polynomial pulse, Gaussian pulse, narrow pulse based on a sinusoidal signal, etc. Gaussian pulse has some unique features, which are good for UWB system. So in this paper, second order derivative of Gaussian pulse is employed as the pulse signal of UWB. The expression of Gaussian pulse is:

$$
g(t)=\frac{A}{\sqrt{2 \pi} \sigma} \exp ^{-\frac{t^{2}}{2 \sigma^{2}}}
$$

Where $A$ is the signal amplitude and is the pulse shaping factor. The expression of second order derivative of Gaussian pulse is:

$$
p(t)=-\frac{A}{\sqrt{2 \pi} \sigma^{3}}\left[1-\left(\frac{t}{\sigma}\right)^{2}\right] e^{-\frac{t^{2}}{2 \sigma^{2}}}
$$

Second order derivative of Gaussian pulse is known as monocycle.

After pulse shaping, the signal undergoes the effects of a channel with $\mathrm{M}$ paths whose response given by:

$$
h(t)=\sum_{m=0}^{M-1} \alpha(m) \delta(t-\tau(m))
$$


Where $\alpha(m)$ and $\tau(m)$ are the gain and delay introduced by the $m^{\text {th }}$ path of the channel.

$$
r(t)=\sum_{i=-\infty}^{\infty} s_{i} \sum_{m=0}^{M-1} \alpha(m) p(t-\tau(m)-i T)+w(t)
$$

Where $\mathrm{T}$ is the symbol rate and $\mathrm{w}(\mathrm{t})$ is additive noise. The noise is assumed to be a zero-mean wide sense stationary process that is uncorrelated with the data, and it may be colored due to narrowband interferers.

In the case of no ISI and when the noise is AWGN, the optimal receiver is a filter matched to the received waveform (i.e. the combined response of the channel and transmit pulse shapes). Typically, this is implemented in a RAKE receiver structure with $\mathrm{L}$ fingers, which can be represented as a filter with response:

$$
f(t)=\sum_{l=0}^{L-1} \beta_{l} p\left(-t-\theta_{l}\right)
$$

Where our model places no restrictions on the spacing of the RAKE delays $\theta_{1}$ and $\beta_{1}$ is the weight of the $\mathrm{m}^{\text {th }}$ finger, the sampled output of the RAKE receiver is then:

$$
y_{n}=[r(t) * f(t)]
$$

From above we get:

$$
y_{n}=\sum_{i=-\infty}^{\infty} s_{i} \sum_{l=0}^{L-1} \sum_{m=0}^{M-1} \alpha(m) \beta(l) R_{p}\left(n T-i T+\theta_{m}-\tau(m)\right)+\breve{w}(T)
$$

Where

$$
\begin{aligned}
& R_{p}(t) \triangleq \int_{-\infty}^{\infty} p(\tau) p(\tau+t) d \tau \\
& R_{p}(t) \triangleq \int_{-\infty}^{\infty} p(\tau) p(\tau+t) d \tau \text { is the time-autocorrelation of the pulse shape }
\end{aligned}
$$

Where

$$
\breve{w}(t)=\sum_{l=0}^{L-1} \beta_{l} \int_{-\infty}^{\infty} w(\tau) p\left(-t+\tau-\theta_{l}\right) d \tau
$$

$\breve{w}(t)=\sum_{l=0}^{L-1} \beta_{l} \int_{-\infty}^{\infty} w(\tau) p\left(-t+\tau-\theta_{l}\right) d \tau$ is the filtered noise. It is well known that the optimal combiner for the AWGN multipath channel is MRC, where $\mathrm{L}=\mathrm{M}$ fingers. When the received signals on each finger are orthogonal (as is the case when there is no ISI), MRC attains the matched filter bound [7]. However, when the ISI becomes significant the orthogonality of the paths is violated unless care is taken in the design of the pulse shape (as in long-coded DS-CDMA systems where successive symbols are nearly orthogonal). For UWB-based high rate WPANs it is anticipated that spreading codes will be short, and therefore MRC is suboptimal even when the noise is AWGN. This motivates a smarter choice of combining weights $\beta_{\mathrm{m}}$, and finger delays $\theta_{\mathrm{m}}$, to combine the signal energy while compensating for the effects of ISI and narrowband interference.

\section{PROPOSED RAKE RECEIVER}

\subsection{Continuous wavelet transform}

Wavelets are defined as small waveforms with different oscillatory structure that is non-zero for a limited period of time (or space). The wavelet transform is a multi-resolution analysis scheme where a signal is decomposed into different frequency components (i.e. at different scales). The wavelet transform basis functions are derived with various continuous scaling and shift parameters, a and $b$ respectively, from the mother wavelet (MW) function $\psi(\mathrm{t})$ as follows:

$$
\psi_{a b}(t)=\frac{1}{\sqrt{a}} \psi\left(\frac{t-b}{a}\right)
$$

There are various MW like, Daubechies wavelets family, Morlet and Mexican Hat. The CWT of the signal $s(t)$ is defined by the wavelet coefficients given by: 


$$
W(a, b)=\int_{-\infty}^{\infty} s(t) \psi_{a b}(t) d t
$$

Where $\mathrm{W}(\mathrm{a}, \mathrm{b})$ represents the similarity between the signal $\mathrm{s}(\mathrm{t})$ and the MR at the scale a and dilation $\mathrm{b}$ (i.e. like the correlation between $\mathrm{s}(\mathrm{t})$ and $\psi_{\mathrm{ab}}(\mathrm{t})$ ). The scale ' $\mathrm{a}$ ' is physically defined as an inverse proportional to the frequency.

\subsection{Proposed rake receiver}

In this proposed adaptive continuous wavelet transform (ACWT) based rake receiver, the continuous wavelet transform of each multipath component of received signal is taken first and is correlated with the template signal or reference signal, which is a continuous wavelet transform of transmitted pulse over different scales. Maximum ratio combiner is used to combine all the fingers output. After the MRC combiner decision logic is applied and data is estimated. Based on the estimated data and the actual desired data, error data is generated and LMS equalizers are used on this error data. The LMS equalizer updates the channel coefficients. Based on these channel coefficients rake parameters such as delay and finger gains are recomputed and updated.

\subsection{Adaptive LMS equalizer}

An adaptive equalizer is an equalizer that automatically adapts to time-varying properties of the communication channel. The most well-known adaptive algorithms are the LMS and the recursive least squares algorithms. Each of them has its own unique properties and applications. In this paper, we use the LMS algorithm due to its simplicity, good stability, and robustness to signal statistics It is a stochastic gradient descent method that uses the gradient vector of the filter tap weights to converge on the optimal Weiner solution.

From the method of steepest descent, the weight vector equation is given by:

$$
w(n+1)=w(n)+\frac{1}{2} \mu\left[-\nabla\left(E\left\{e^{2}(n)\right\}\right)\right]
$$

Where $\mu$ is the step-size parameter and controls the convergence characteristics of the LMS algorithm, $e^{2}(n)$ is the mean square error between the output $y(n)$ and the reference signal which is given by,

$$
e^{2}(n)=[d(n)-\hat{d}(n)]^{2}
$$

In order to achieve a fast initial convergence speed and to retain a fast tracking ability in the steady state, large value for step size is chosen. On the other hand, large step size will result in large steady maladjustment error.

\section{SIMULATION RESULTS AND ANALYSIS}

This section presents the simulation results and the performance analysis of the ACWT rake receiver over the conventional rake receiver and the CWT rake receiver. For simulation purpose we have chosen the transmitted pulse as Gaussian $2^{\text {nd }}$ order derivate pulse of pulse width $0.5 \mathrm{~ns}$ with a sampling period of $0.05 \mathrm{~ns}$. Frame time of 20ns seconds is selected with a constrain such that one pulse per frame.

UWB channels CM1, CM2, CM3 and CM4 with a channel impulse response of 0.5 are used as the medium for transmission of data. Initial training data of 100 bits is given to system for the channel estimation purpose.

Figure 1, Figure 2 and Figure 3 shows the performance of the adaptive CWT rake receiver with the conventional rake receiver and the CWT rake receiver for CM1 UWB channel with $\mathrm{L}=2,4,8$ respectively.

For UWB channel CM1, Figure 1 shows that when the number of rake fingers (L) equal to 2, in adaptive continuous wavelet rake receiver, BER of $10^{-3}$ is achieved at a very low SNR i.e. at $13 \mathrm{~dB}$ where as in continuous wavelet rake receiver we achieve the same BER at SNR of $16 \mathrm{~dB}$ and in convention we achieve at very high SNR compared to adaptive CWT and CWT rake receivers. As the number of rake fingers increases, there is an improvement in the performance of both CWT and adaptive CWT rake receiver as seen in the Figure 2 and Figure 3 .For $\mathrm{L}=4$ and 8 performance of proposed rake receiver is $3 \mathrm{~dB}$ and $2 \mathrm{~dB}$ superior to that of the CWT rake receiver. 


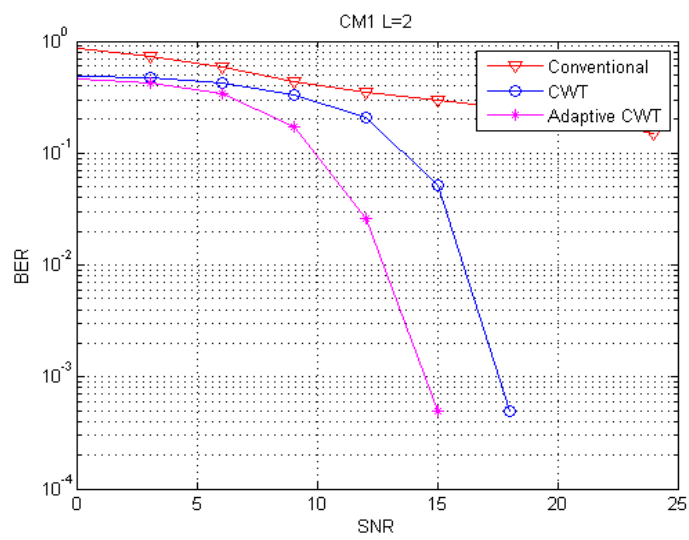

Figure 1. BER performance analysis for selective rake receiver with $\mathrm{CM} 1$ and $\mathrm{L}=2$

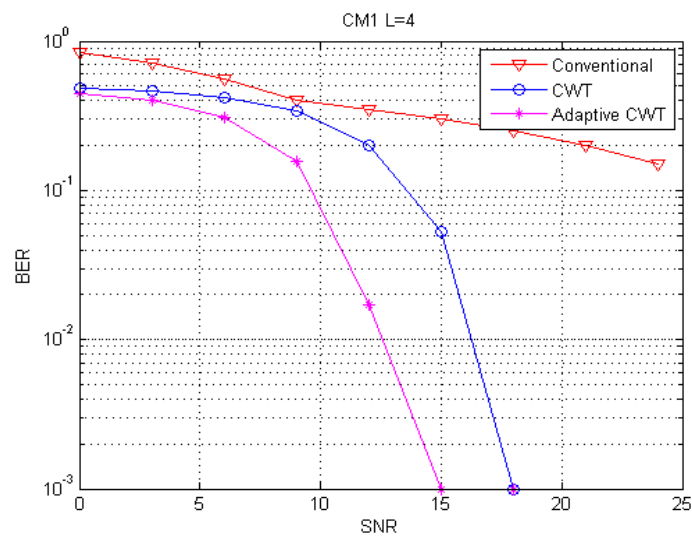

Figure 2. BER performance analysis for selective rake receiver with $\mathrm{CM} 1$ and $\mathrm{L}=4$

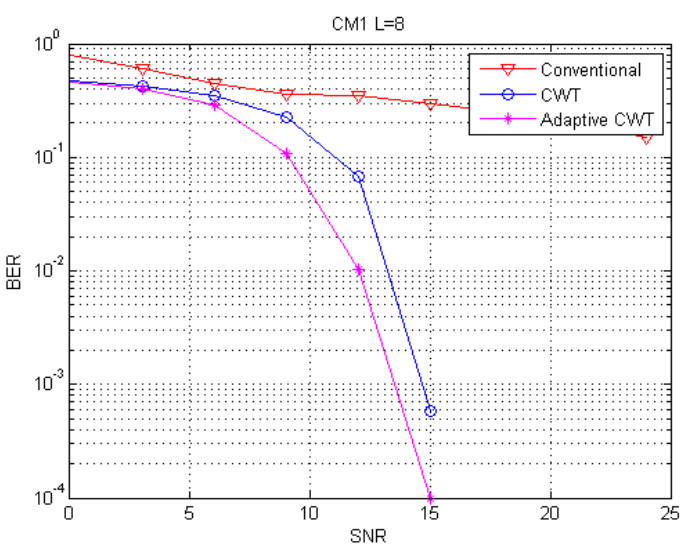

Figure 3. BER performance analysis for selective rake receiver with $\mathrm{CM} 1$ and $\mathrm{L}=8$

Figure 4, Figure 5 and Figure 6 shows the performance of the adaptive CWT rake receiver with the conventional rake receiver and the CWT rake receiver for CM2 UWB channel with L=2, 4 and 8 respectively.

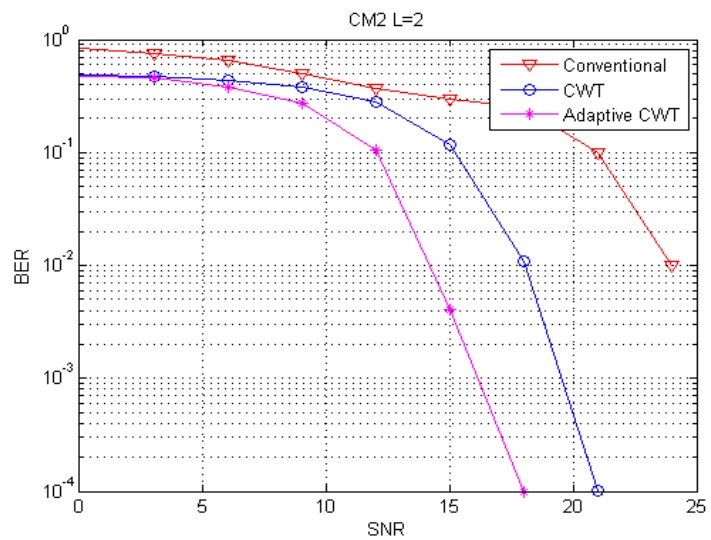

Figure 4. BER performance analysis for selective rake receiver with $\mathrm{CM} 2$ and $\mathrm{L}=2$

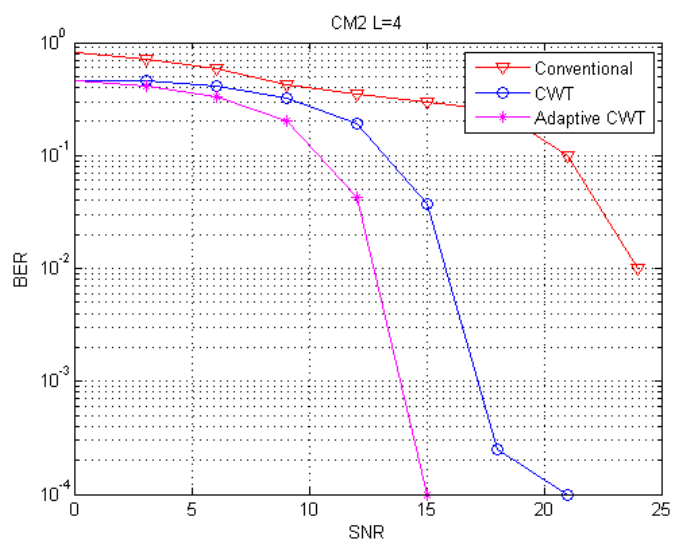

Figure 5. BER performance analysis for selective rake receiver with $\mathrm{CM} 2$ and $\mathrm{L}=4$ 


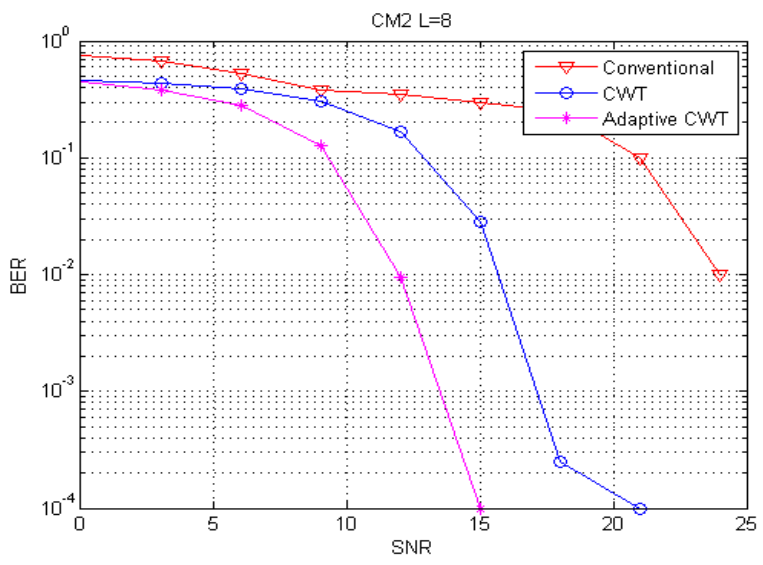

Figure 6: BER performance analysis for selective rake receiver with $\mathrm{CM} 2$ and $\mathrm{L}=8$

For UWB channel CM2, Figure 4 shows that when the number of rake fingers (L) equal to 2, in adaptive continuous wavelet rake receiver BER equal to $10^{-3}$ is achieved at a very low SNR i.e. at $16 \mathrm{~dB}$ where as in continuous wavelet rake receiver (CWT) we achieve the same BER at SNR of $19 \mathrm{~dB}$ and in convention we achieve at very high SNR compared to adaptive CWT and CWT rake receivers. As the number of rake fingers increases, there is an improvement in the performance of both CWT and adaptive CWT rake receiver as seen in the Figure 5 and Figure 6. For $\mathrm{L}=4$ and 8 performance of proposed rake receiver is $3 \mathrm{~dB}$ and $3 \mathrm{~dB}$ superior to that of the CWT rake receiver.

Figure 7, Figure 8 and Figure 9 shows the performance of the adaptive CWT rake receiver with the conventional rake receiver and the CWT rake receiver for CM3 UWB channel with L=2, 4 and 8 respectively.

For UWB channel CM3, Figure 7 shows that when the number of rake fingers (L) equal to 2, in adaptive continuous wavelet rake receiver, BER of $10^{-3}$ is achieved at a very low SNR i.e. at $18 \mathrm{~dB}$ where as in continuous wavelet rake receiver we achieve the same BER at SNR of $28 \mathrm{~dB}$ and in convention we achieve at very high SNR compared to adaptive CWT and CWT rake receivers. As the number of rake fingers increases, there is an improvement in the performance of both CWT and adaptive CWT rake receiver as seen in the Figure 8 and Figure 9. For $\mathrm{L}=4$ and 8 performance of proposed rake receiver is $10 \mathrm{~dB}$ and $10 \mathrm{~dB}$ superior to that of the CWT rake receiver.

Figure 10, Figure 11 and Figure 12 shows the performance of the adaptive CWT rake receiver with the conventional rake receiver and the CWT rake receiver for CM4 UWB channel with $\mathrm{L}=2,4$ and 8 respectively.

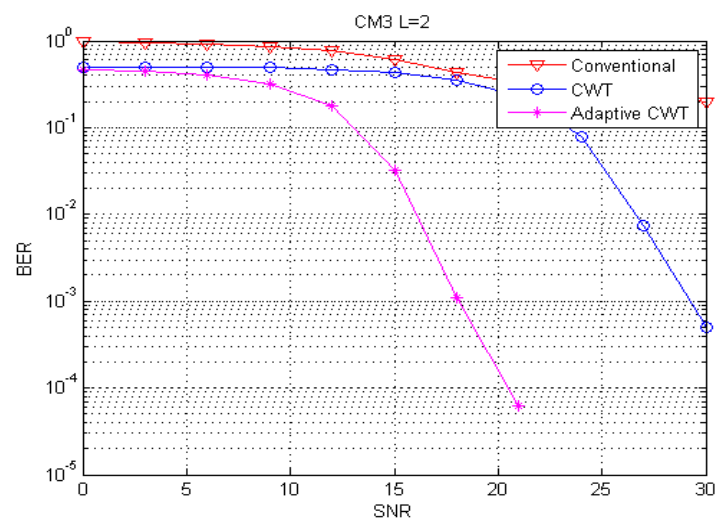

Figure 7. BER performance analysis for selective rake receiver with $\mathrm{CM} 3$ and $\mathrm{L}=2$

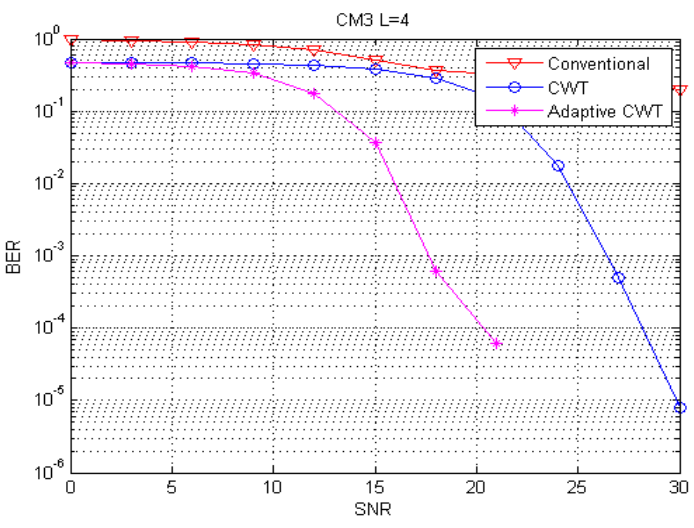

Figure 8. BER performance analysis for selective rake receiver with $\mathrm{CM} 3$ and $\mathrm{L}=4$ 


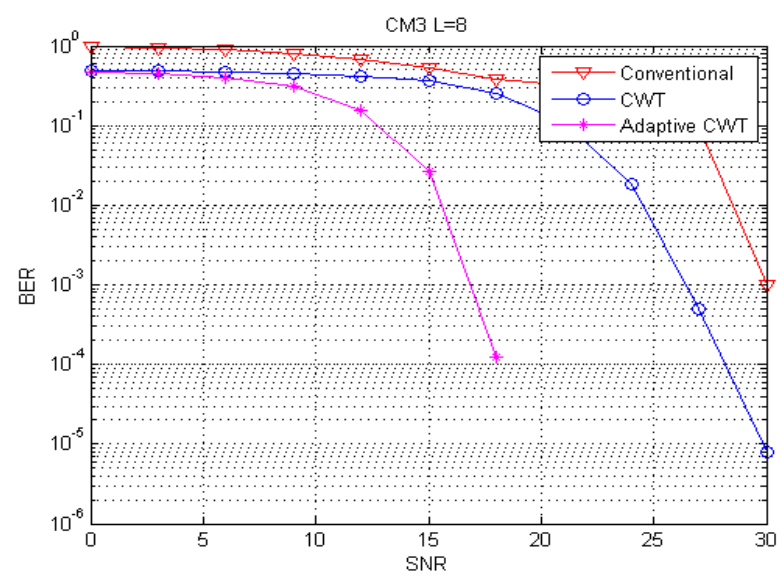

Figure 9. BER performance analysis for selective rake receiver with $\mathrm{CM} 3$ and $\mathrm{L}=8$

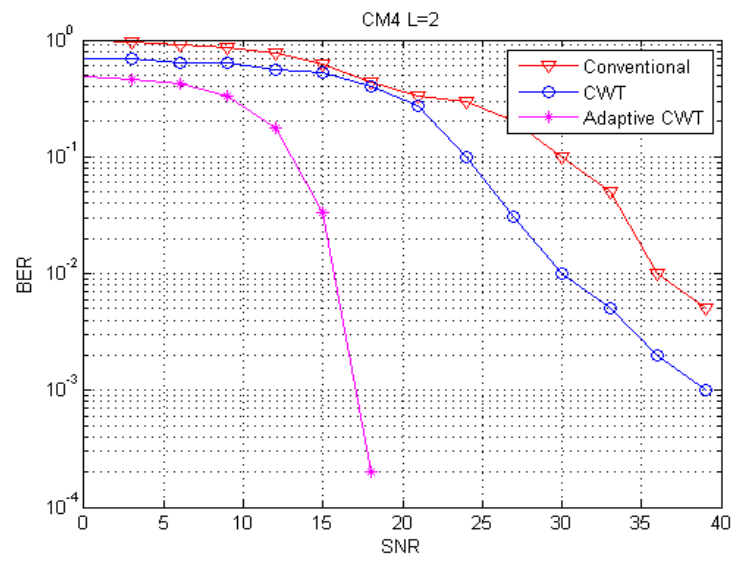

Figure 10. BER performance analysis for selective rake receiver with $\mathrm{CM} 4$ and $\mathrm{L}=2$

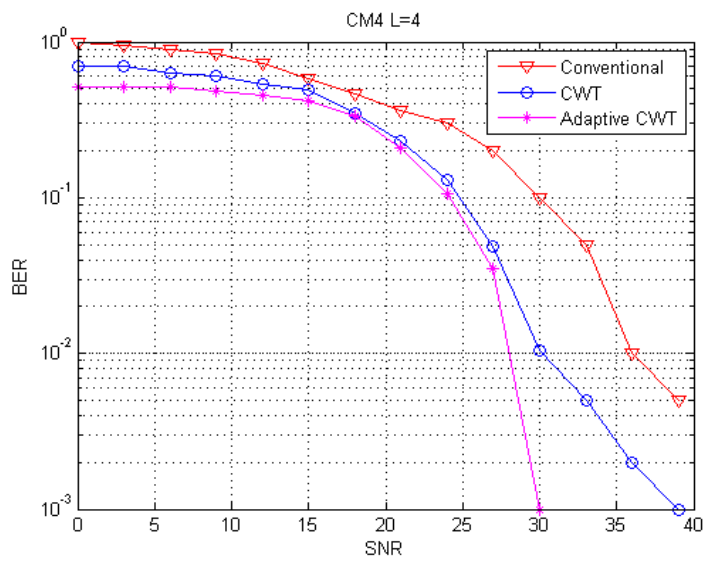

Figure 11. BER performance analysis for selective rake receiver with $\mathrm{CM} 4$ and $\mathrm{L}=4$

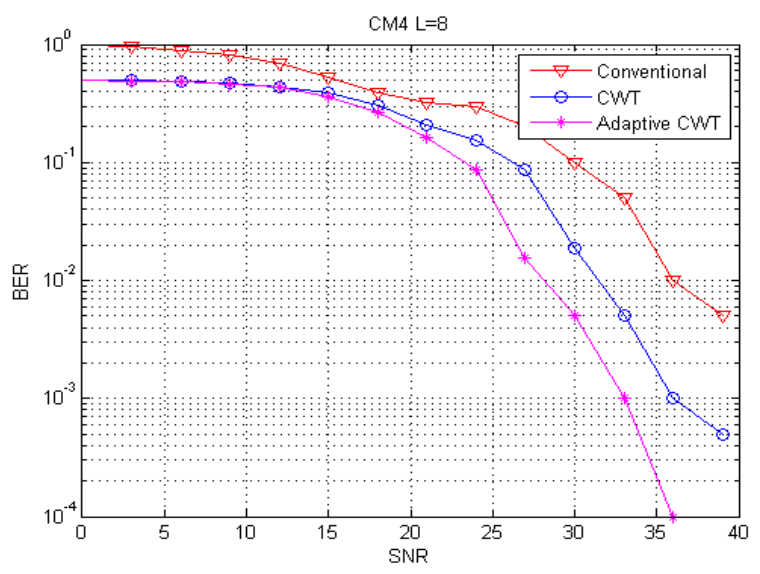

Figure 12. BER performance analysis for selective rake receiver with $\mathrm{CM} 4$ and $\mathrm{L}=8$

For UWB channel CM4, Figure 10 shows that when the number of rake fingers (L) equal to 2, in adaptive continuous wavelet rake receiver, BER of $10^{-3}$ is achieved at a very low SNR i.e. at $17 \mathrm{~dB}$ where as 
in continuous wavelet rake receiver we achieve the same BER at SNR of $36 \mathrm{~dB}$ and in convention we achieve at very high SNR compared to adaptive CWT and CWT rake receivers. As the number of rake fingers increases, there is an improvement in the performance of both CWT and adaptive CWT rake receiver as seen in the figure 11 and 12 .For $\mathrm{L}=4$ and 8 performance of proposed rake receiver is $3 \mathrm{~dB}$ and $2 \mathrm{~dB}$ superior to that of the CWT rake receiver.

\section{CONCLUSION}

The huge bandwidth occupancy of the UWB system led to the development of the CWT rake receivers. Based on this, a fully adaptive CWT rake receiver was designed and implemented in this paper. As the channel is considered to be blind, a training based channel estimation and a LMS equalizer for delay and weights adjustment is studied and implemented. On comparing, the adaptive CWT rake receiver, CWT rake receiver and conventional rake receiver with 2, 4 and 8 rake fingers of UWB channel models one to four (CM1 to CM4), we conclude that adaptive CWT rake receiver has shown better performance compared to remaining rake receivers. Simulations showed that for 8 rake fingers, the proposed adaptive CWT rake receiver has shown an SNR improvement of $2 \mathrm{~dB}, 3 \mathrm{~dB}, 10 \mathrm{~dB}$ and $2 \mathrm{~dB}$ respectively over CWT rake receiver in different UWB channels CM1, CM2, CM3 and CM4.

\section{REFERENCES}

[1] Razali Ngah and Yasser Zahedi, "UWB Communications: Present and Future”, IEEE Asia-Pacific Conference on Applied Electromagnetics (APACE), 2016, pp373-378.

[2] Vikas Goyal and B. S. Dhaliwal, "Optimal Pulse Generation for the Improvement of Ultra Wideband System Performance", Int. Conf. Recent Advances in Engineering and Computational Sciences, IEEE Explore digital library with, ISBN: 978-1-4799-2291-8 held at UIET, Punjab University, Chandigarh, March 6-8, 2014.

[3] Tabaa, M. and Diou, C., "A Low-Cost Many-to-One WSN Architecture Based on UWB-IR and DWPT", International conference on Control, Decision and Information technologies CoDIT2014 IEEE, Metz, pp. 3-5, Nov. 2014.

[4] Chehaitly, et al., "A Low-Cost M. Tabaa 208 Design of Tranceiver Based on DWPT for WSN", International Conference on Microelectronics ICM2015, Casablanca, pp. 20-23, Dec. 2015.

[5] L. Stoica, et al., "An Ultrawideband System Architecture for tag based Wireless Sensor Networks", IEEE Trans. Veh. Technol., vol. 54, pp. 1632-1645, 2005.

[6] L. Y. Astanin and A. A. Kostylev, "Ultra wideband Radar Measurements Analysis and Processing", London U.K.: IEE, 1997.

[7] "Revision of Part 15 of the Commission's Rules Regarding Ultra-Wideband Transmission Federal Communications Commission", 1st Rep. and Order, 2002.

[8] T. W. Barrett, "History of Ultra-wideband Communications and Radar: Part I, UWB Communications", Microw. J., pp. 22-56, Jan. 2001.

[9] M. Z. Win and R. A. Scholtz, "Impulse Radio: How it works", IEEE Commun. Lett., vol. 2, pp. 36-38, Feb. 1998.

[10] M. Z. Win and R. A. Scholtz, "Ultra-wide Bandwidth Time-hopping Spread-spectrum Impulse Radio for Wireless Multiple-access Communications", IEEE Trans. Commun., vol. 48, pp. 679-689, Apr. 2000.

[11] F. Ramirez-Mireles, "Performance of Ultrawideband SSMA using Time Hopping andM-ary PPM", IEEE J. Select. Areas Commun., vol. 19, pp. 1186-1196, 2001.

[12] R. T. Hoctor and H.W. Tomlinson, "An Overviewof Delay-hopped, Transmitted Reference RF Communications", in Technical Information Series: G.E. Research and Development Center, Jan. 2002, pp. 1-29.

[13] J. R. Foerster, "The Performance of a Direct Sequence Spread Ultra-wideband System in the Presence of Multipath, Narrowband Interference, and Multiuser Interference", in Proc. 2002 UWBST, 2002, pp. 87-92.

[14] Hua Shao; Norman C.Beaulieu, "Direct sequence and time hopping sequence designs for narrowband interference mitigation in impulse radio UWB systems", IEEE Transactions on Communications, vol. 59, no. 7, 2011, pp1957-1965.

[15] J. D. Choi and W. E. Stark, "Performance Analysis of Ultra-wideband Spread-spectrum Communications in Narrowband Interference", in Proc. 2002 MILCOM, 2002.

[16] John D. Choi, and Wayne E. Stark "Performance of Ultra-Wideband Communications with Suboptimal Receivers in Multipath Channels", IEEE Journal on Selected Areas in Communications, vol. 20, no. 9, December 2002.

[17] Boukrouche Abdelhani, "Performance Analysis of RAKE Receiver for TH-UWB Systems", The International Conference on Embedded Systems in Telecommunications and Instrumentation (ICESTI'16), At Annaba, Algeria, October, pp. 24-26, 2016.

[18] B. Mielczarek, et al., "Performance of Coherent UWB Rake Receivers with Channel Estimators", IEEE 58th, Vehicular Technology Conference, vol. 3, October 2003.

[19] Oppermann, et al., "UWB Theory and Applications", England, John Wiley \& Sons, Ltd, 2004.

\footnotetext{
Performance Evaluation of Adaptive Continuous Wavelet Transform based Rake Receiver ... (Ch. Navitha)
} 
[20] M. Z. Win, G. Chrisikos, N. R. Sollenberger, "Performance of Rake Reception in Dense Multipath Channels: Implications of Spreading Bandwidth and Selection Diversity Order", IEEE Journal on Selected Areas in Communications, vol. 18, no. 8, August 2000.

[21] Said E. El-Khamy, Ehab F. Badran, Amira I. Zaki "UWB Analog Space Time Coding Systems Using A Genetic Algorithm Based Adaptive Rake Receiver", Proceedings of the 4thInternational Conference on Signal Processing and Communication Systems, Australia, December 2010.

[22] Mahboob Iqbal, et al., "SAR Image Despeckling by Selective 3D Filtering of Multiple Compressive Reconstruction Images", Progress In Electromegnatic Research, vol. 134, 2013, pp. 209-226.

[23] Minh Hung Le and Nikos E. Mastorakis, "Performance Analysis of Wideband MCCDMA for Wavelet Vidio with Multilevel UEP Code over Fading Channels", 5th WSEAS INT Conf. Corfu, Greece, Aug. 2005, pp 114-119.

[24] Amira I. Zaki, Said E. El-Khamy, Ehab F. Badran. A Novel Rake Receiver Based on Continuous Wavelet Transform Designed for UWB Systems. in European Journal of Scientific Research • August 2012

[25] Ch. Navitha, et al., "Use of Dual Tree Complex Wavelet Transform for UWB system RAKE receiver", International Conference on Control, Instrumentation, Communication and Computational Technologies (ICCICCT), $16^{\text {th }}$ and $17^{\text {th }}$ December 2016, pp. 668-671.

[26] G. Fock, et al., "Channel Tracking for RAKE Receivers in Closely Spaced Multipath Environments", IEEE Journal on selected areas in communication, vol. 19, no. 12, December 2001, pp. 2420-2431.

[27] J. Foerster, et al., "A Channel Model for Ultra-wideband Indoor Communications", Proceedings of the 6th International Symposium on Wireless Personal Multimedia Communications, Yokosuka, Japan, pp. 116-120, 2003.

[28] L. Yang and G. B. Giannakis, "Analog Space-Time Coding for Multi-antenna Ultra-Wideband Transmissions", IEEE Transactions on Communications, vol. 52, no. 3, March 2004. 